\title{
Prognostic Value of Preoperative Inflammation-Based Prognostic Scores on Hospital Mortality after Gastric Cancer Surgery
}

\author{
Tolga Dinc ${ }^{1}$, Baris Dogu Yildiz ${ }^{1 *}$, Ilgaz Kayilioglu ${ }^{1}$, Isa Sozen ${ }^{1}$, Mesut Tez ${ }^{1}$, Faruk \\ Coskun ${ }^{2}$
}

\begin{abstract}
Gastric cancer is the fourth most common malignancy worldwide. Poor prognosis in gastric cancer is linked with hypoalbuminemia. Previous studies demonstrated relationships between gastric cancer and chronic inflammation. The aim of this study was to assess the prognostic value of preoperative inflammation-based prognostic scores regarding hospital mortality after gastrectomy. Unlike other recent articles on the same topic; we could only verify prognostic value of prognostic nutritional index for in hospital mortality after surgery.
\end{abstract}

Keywords: Gastric cancer - prognostic score - mortality - prognostic nutritional index - NLR - platelet lymphocyte ratio

Asian Pac J Cancer Prev, 15 (18), 7909-7911

\section{Introduction}

The presence of an inflammatory response is crucial in the development of cancer-associated malnutrition, resulting in poor performance status and increased mortality in patients with cancer. On the basis of the relationship between inflammation and cancer progression, several inflammation-based scores have been proposed as prognostic. The main scores that have been associated with cancer-specific survival are the modified Glasgow Prognostic Scores, neutrophil lymphocyte ratio (NLR), platelet lymphocyte ratio (PLR) and prognostic nutritional index (PNI) (Pinato 2012; Bugada 2014).

To our knowledge, there are not any studies elucidating which of these prognostic scores is more suitable in predicting outcomes in patients with gastric cancer. The aim of this study was to compare the prognostic value of these preoperative inflammation based prognostic scores (NLR, PLR and PNI) in hospital mortality after gastrectomy.

\section{Materials and Methods}

This is a retrospective clinical study involving 147 patients who had surgery for gastric cancer between January 2012 and October 2013 at Ankara Numune Teaching Hospital General Surgery Clinic. All patients had gastrectomy (total/subtotal) and D2 lymph node dissection. Data was collected from patient files and included demographic variables, type of surgery, histopathological diagnosis, serum albumin level, complete blood count and survival on follow up.

NLR, PLR, PNI were calculated based on the equations shown on Table 1. Patients with incomplete data, those who received neoadjuvant chemotherapy and those with distant metastasis were excluded from study. Patients who died in the first 30 days after surgery were accepted as hospital mortality.

The end-point of our study was relation between NLR, PLR, PNI and hospital mortality.

\section{Results}

Fourty-six (31.3\%) patients were female and 101 $(68.7 \%)$ were male. Mean age was $63.87 \pm 11.01$ years. $97(66 \%)$ patients were alive at the time of study and $40(44 \%)$ were dead. 15 of the deceased patients died within 30 days after surgery thus these were classified as hospital mortality (Group 1). The remaining 132 patients were classified as Group 2. Table 2 shows age and gender

Table 1. Formulas for Calculation of Neutrophil Lymphocyte Ratio, Platelet Lymphocyte Ratio and Prognostic Nutrition Index

Neutrophil lymphocyte ratio

Neutrophil count / lymphocyte count

Platelet lymphocyte ratio

Platelet count / lymphocyte count

Prognostic nutrition index

Albumin (g/dl)+ X total lymphocyte count X 109/1 
Table 2. Age and Gender Distribution of Groups

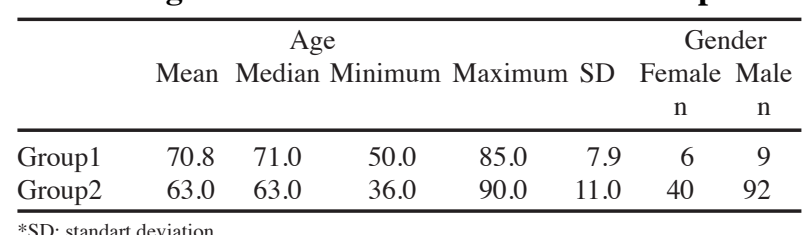

*SD: standart deviatio

Table 3. Comparison of Scores in Patient Groups

\begin{tabular}{llrrccc}
\hline Score & Group & $\mathrm{n}$ & Mean & $\begin{array}{c}\text { Std. } \\
\text { Deviation }\end{array}$ & $\begin{array}{c}\text { Std. } \\
\text { Error Mean }\end{array}$ & $\mathrm{p}$ \\
\hline NLR & Group-1 & 15 & 5.9 & 5.0 & 1.3 & 0.28 \\
& Group-2 & 132 & 4.2 & 5.2 & 0.5 & \\
PNI & Group-1 & 15 & 43.9 & 11.00 & 2.84 & 0.008 \\
& Group-2 & 132 & 48.0 & 7.76 & 0.70 & \\
PLR & Group-1 & 15 & 225.1 & 168.1 & 43.4 & 0.60 \\
& Group-2 & 132 & 202.5 & 165.6 & 14.9 & \\
\hline
\end{tabular}

${ }^{*} \mathrm{p}<0.05$ is set as significant; NLR: neutrophil lymphocyte ratio; PNI: prognostic nutrition index; PLR: platelet lymphocyte ratio

distribution of the two groups. Mean PNI was calculated as $43.89 \pm 11.00$ in Group 1 and $48.02 \pm 7.76$ in Group 2 . This difference was statistically significant $(\mathrm{p}=0.008)$ revealing association between in hospital mortality and PNI. There was not a statistically significant difference between groups in terms of NLR and PLR ( $p>0.05)$. Table 3 summarizes calculated values of NLR, PLR, PNI in each group.

\section{Discussion}

Apart from cancer related factors, evidence shows that systemic inflammatory response of the host is also an integral part of outcome in oncological patients (QiMing.,2012). Initially inflammatory response is directed at destroying the insult but when this response becomes persistent it starts helping the advance of the disease such as cancer. Numerous studies proposed assessment of prognostic factors for survival in patients with cancer and some of them focused on inflammatory markers. Ongoing inflammation causes loss of vital proteins such as albumin and changes levels of circulating white blood cells (increase in neutrophils and decrease of lymphocytes) in cancer (Hanahan and Weinberg, 2011).

Nutritional impairment is correlated with poor performance status, shorter survival and increased mortality in patients with cancer (Andreyev et al, 1998). Albumin is an indicator of nutrition correlating with postoperative complications. Low albumin levels are shown to be secondary to sustained inflammatory response incited by the host.( Fujiwara et al., 2010)

PNI was initially designed to assess the immunological and nutritional aspects of patients undergoing surgery of the gastrointesintal tract. (Kanda et al., 2011) Later PNI has been shown to be a prognostic marker in a number of malignancies regardless of the site of origin (Proctor et al, 2011). In our study, we showed that PNI was related to 30 day mortality after gastric cancer surgery.

Recent studies have shown that the relationship between neutrophilia and lymphopenia (ie NLR) is an independent prognostic factor in gastric cancer (Jung et al, 2011). NLR is a measure of inflammation based on standard laboratory measurements of total lymphocyte count and neutrophil count which are routinely measured in the clinical setting. Recent data showed that preoperative NLR could be a prognostic marker in patients with gastric cancer (Ma 2011; Cho 2014). But, we did not verify this for 30 day mortality after gastric cancer surgery in our study. The same was true for PLR as our study could not find a relation between 30 day mortality and PLR in contrary to recent findings of other authors (Lee 2013; Jiang 2014).

The two shortcomings of our study are retrospective design and fairly low size of our groups. Our next research will be testing the same hypothesis as a prospective design.

In conclusion, various studies investigated a number of prognostic factors in gastric cancer. In the present study we found that PNI was superior to the presence of NLR and PLR in predicting 30 day mortality of a cohort of patients with gastric cancer. This could be very valuable in the management of this patient population as treatment decisions can be directed using the preoperatively calculated PNI based on routine laboratory measures. Thus PNI could be evaluated not only as a prognostic marker in patients with gastric cancer but also for grouping patients in planning the proper treatment approach.

\section{References}

Andreyev HJ, Norman AR, Oates J, Cunningham D (1998). Why do patients with weight loss have a worse outcome when undergoing chemotherapy for gastrointestinal malignancies? Eur J Cancer, 34, 503-9.

Bugada D, Allegri M, Lavand'homme P, De Kock M, Fanelli G (2014). Inflammation-Based Scores: A New Method for Patient-Targeted Strategies and Improved Perioperative Outcome in Cancer Patients. Biomed Res Int, 2014, 1424-5.

Cho IR, Park JC, Park CH, et al (2014). Pre-treatment neutrophil to lymphocyte ratio as a prognostic marker to predict chemotherapeutic response and survival outcomes in metastatic advanced gastric cancer. Gastric Cancer, 19.

Crumley AB, Stuart RC, McKernan M, McMillan DC (2010). Is hypoalbuminemia an independent prognostic factor in patients with gastric cancer? World J Surg, 34, 2393-8.

Fujiwara Y, Shiba H, Furukawa K, et al (2010). Glasgow prognostic score is related to blood transfusion requirements and post-operative complications in hepatic resection for hepatocellular carcinoma. Anticancer Re, 30, 5129-6.

Hanahan D, Weinberg RA (2011). Hallmarks of cancer: the next generation. Cell, 144, 646-74.

Jiang N, Deng JY, Liu Y, et al (2014). The role of preoperative neutrophil-lymphocyte and platelet-lymphocyte ratio in patients after radical resection for gastric cancer. Biomarkers, 9, 1-8.

Jung MR, Park YK, Jeong O, et al (2011). Elevated preoperative neutrophil to lymphocyte ratio predicts poor survival following resection in late stage gastric cancer.J Surg Oncol, 104, 504-10.

Kanda M, Fujii T, Kodera Y, et al (2011). Nutritional predictors of postoperative outcome in pancreatic cancer. Br J Surg, 98, 268-4.

Lee S, Oh SY, Kim SH, et al (2013). Prognostic significance of neutrophil lymphocyte ratio and platelet lymphocyte ratio in advanced gastric cancer patients treated with FOLFOX chemotherapy. BMC Cancer, 22, 13-350.

Lin WL, Sun JL, Chang SC, et al (2014). Factors predicting 
survival of patients with gastric cancer. Asian Pac J Cancer Prev, 15, 5835-8.

Ma JP, Wang Z, Lin JW, et al ( 2011). Neutrophil-lymphocyte ratio as a prognostic factor in gastric cancer. Zhonghua Wei Chang Wai Ke Za Zhi, 14, 944-7.

Pinato DJ, North BV, Sharma R (2012). A novel, externally validated inflammation-based prognostic algorithm in hepatocellular carcinoma: the prognostic nutritional index (PNI). Br J Cancer, 106, 1439-45.

Proctor MJ, Morrison DS, Talwar D, et al (2011). A comparison of inflammation-based prognostic scores in patients with cancer. A Glasgow Inflammation Outcome Study. Eur J Cancer, 47, 2633-41.

Qi-Ming Yu, Chuan-Ding Yu, Zhi-Qiang Ling (2012). Elevated circulating CD19 lymphocytes predict survival advantage in patients with gastric cancer. Asian Pacific J Cancer Prev, 13, 2219-4.

Qing-Qing Li, Zhi-Hao Lu, Li Yang, et al (2014). Neutrophil count and the inflammation-based glasgow prognostic score predict survival in patients with advanced gastric cancer receiving first-line chemotherapy. Asian Pac JCancer Prev, 15, 945-50. 